\title{
TTR
}

Traduction, terminologie, re?daction

\section{La réception de l'essai sur la traduction dans le domaine français}

\section{Alexis Nouss}

Volume 10, numéro 2, 2e semestre 1997

L'essai sur la traduction de Walter Benjamin : traductions critiques Walter Benjamin's Essay on Translation: Critical Translations

URI : https://id.erudit.org/iderudit/037300ar

DOI : https://doi.org/10.7202/037300ar

Aller au sommaire du numéro

\section{Éditeur(s)}

Association canadienne de traductologie

ISSN

0835-8443 (imprimé)

1708-2188 (numérique)

Découvrir la revue

Citer cet article

Nouss, A. (1997). La réception de l'essai sur la traduction dans le domaine français. TTR, 10(2), 71-85. https://doi.org/10.7202/037300ar

\section{Résumé de l'article}

La réception de l'essai sur la traduction dans le domaine français - Cette recension critique rend compte d'une dizaine de titres ayant traité de l'essai sur la traduction de Walter Benjamin afin d'en présenter les grandes lignes de la réception dans le domaine français. L'auteur y montre comment, sauf exceptions, l'essai est souvent lu de manière partiale, restrictive ou coupé du reste de l'oeuvre. 


\title{
La réception de l'essai sur la traduction dans le domaine français
}

\author{
Alexis Nouss
}

La parution des deux volumes d'œuvres choisies de Walter Benjamin traduites et préfacées par Maurice de Gandillac ( 1 . Mythe et violence, 2. Poésie et révolution, Paris, Denoël, 1971) introduit l'essai, figurant au premier tome, dans le domaine français. C'est à la même période qu'il est offert au lectorat anglophone dans le recueil Illuminations (New York, Schocken Books, 1969), traduit par Harry Zohn, édité et préfacé par Hannah Arendt.

Le lien de Benjamin à la France n'est pas fortuit. Très tôt, il commence à commenter et traduire la littérature française, arpente la scène intellectuelle parisienne, et la capitale, où il séjourne fréquemment, deviendra le matériau de son travail critique et historique sur la modernité avant d'accueillir son exil, fuyant le nazisme. Dès les années cinquante, paraissent des articles sur son cuvre et les années soixante-dix l'intronisent, non sans ambiguilté et parfois en toute méconnaissance, au panthéon des grandes figures mythico-intellectuelles. N'oublions pas, en outre, que l'essai introduit une traduction de Baudelaire. 
Pour la traductologie d'expression française, dans l'agitation discursive de ses institutions (publications spécialisées, recherches universitaires, colloques et congrès...) l'essai est devenu référence récurrente, souvent cité - une phrase ou une expression hors contexte -, rarement étudié dans la cohésion de ses articulations internes et la cohérence avec le reste de l'œuvre critique ou philosophique, offrant davantage une caution ou une légitimation qu'une argumentation. À ce qu'en disait Paul de Man : « [...] dans la profession vous ne pouviez vous faire un nom à moins d'avoir dit quelque chose sur ce texte. ( $(1991$, p. 23) fait écho la remarque de Jean-René Ladmiral : " Dans le domaine de la traduction, il arrive que le nom de Walter Benjamin fonctionne comme une manière de schibboleth [...]. * $(1981$, p. 67) Quelques vagues idées tenant lieu d'interprétation, la lecture courante réduit fréquemment le contenu du texte à une défense rigide du littéralisme et à la revendication mystique d'une langue originelle, deux notions certes présentes mais articulées avec une grande complexité conceptuelle. Sans vouloir analyser ici en profondeur les raisons de cette compréhension fallacieuse et lacunaire, une cause apparait dominante : si, en matière de théorie de la traduction, désormais pluraliste, l'approche philosophique est admise, à côté des perspectives linguistique, sémiotique ou sociologique, la rigueur et la connaissance qu'elle suppose sont négligées. Or l'essai de Benjamin est proprement philosophique et, à ce titre, demande à être situé, y compris en traductologie, à la fois dans l'histoire de la philosophie ${ }^{1}$ et dans l'horizon global de la pensée benjaminienne.

${ }^{1}$ Sur ce point, la réception de Benjamin n'a pas manqué de souffrir de l'horizon heideggerien dans lequel s'est complu pendant des décennies un courant important de la philosophie d'expression française, notamment d'obédience phénoménologique (avec des retombées, bien évidemment, dans l'ensemble du champ des sciences humaines). Quant à la question du langage, les deux pensées sont souvent rapprochées alors que leur confrontation antagoniste s'avère bien plus féconde. Voir à titre d'exemple l'article de L. Lamy dans le présent numéro. 
Peut-être faudrait-il aussi interroger une certaine frilosité du domaine français, repérable dans d'autres disciplines, à l'égard des pensées non-systématiques. On goûte fort les " ismes " globalisateurs, dussent-ils se succéder au gré des vents idéologiques, en se méfiant des esprits traversiers ou vagabonds. Mais, par définition, la traduction ne les requiert-elle pas?

Un certain nombre de textes de valeur ont néamnoins été consacrés à l'essai et si leur nombre est moins important que dans le domaine anglophone, il semble des plus utiles d'en proposer une recension critique. Avec la seule ambition de ne livrer qu'un bref aperçu, nous ne nous sommes pas livré à une étude chronologique, abandonnant également toute prétention à l'exhaustivité, et avons préféré dégager divers courants ou territoires interprétatifs en choisissant et regroupant quelques lectures jugées représentatives. Celles-ci émanent soit de théoriciens figurant dans un corpus traductologique reconnu, soit d'auteurs approchant Benjamin dans une perspective plus spécifiquement philosophique.

Avec des exceptions, cette distinction recouvre la complémentarité du présent article et de celui qui suit, dû à Laurent Lamy².

Le rôle incontestable joué par George Steiner dans la réception de Benjamin en français est cependant ambivalent. Il faut sans doute indiquer au préalable que la parution de son Après

\footnotetext{
${ }^{2}$ Pour des raisons évidentes, nows ne rendons pas compte des articles que L. Lamy et moi-même avons signés dans le cadre d'une recherche commune. Nous les indiquons cependant parmi les références et y renvoyons le lecteur.
} 
Babel en 1978, trois ans après celle de After Babel, revêtit une importance considérable dans le développement de la traductologie en ce qu'il accordait une dignité épistémologique à la traduction en en faisant le paradigme dynamique de toute communication humaine. Par ailleurs l'ouvrage par son ampleur - sa visée réflexive, l'érudition déployée, la diversité culturelle et linguistique des sources $\rightarrow$ demeure indispensable dans le corpus de base de la discipline. Or, dans cette révaluation, Steiner s'appuie explicitement sur Benjamin qu'il cite parmi la dizaine de noms qui ont fondé la théorie de la traduction et dont la référence est constante au long des pages. Mais sa lecture est très orientée et donne de la pensée benjaminienne une catégorisation étroite. Benjamin, aux côtés de Kafka et Borges, illustre le courant moderne de ce que Steiner nomme la tradition gnostique et est rattaché à la spéculation ésotérique, plus spécifiquement à la mystique juive (1978, p. 70 sq.; p. 231), même si, ailleurs, Steiner situe Benjamin dans la phase herméneutique de la théorie de la traduction (p. 225) et même s'il identifie théologie et philosophie (p. 258, n. 31). Cette approche s'avère réductrice et peut mener au contre-sens lorsque la " reine Sprache ", le pur langage, est compris comme a langue universelle " première et sous-jacente aux diverses manifestations linguistiques. Dans une dérive métaphysique tendant à valoriser la recherche d'une transcendance ou d'un absolu, l'insistance de Benjamin sur l'historicité des pratiques langagières est ignorée au profit d'une analyse dont l'inspiration heideggerienne est manifeste et avouée.

C'est en revanche sous les ailes de l'Ange de l'histoire que Stéphane Mosès livre sa lecture groupée des ceuvres de Rosenzweig, Benjamin et Scholem. Comme l'indique le titre, l'emprunt de catégories théologiques permet à ces auteurs de renouveler une philosophie de l'histoire déficiente et impuissante à saisir les désastres du XX' ${ }^{\mathrm{e}}$ siècle en l'énonçant dans les termes d'une dynamique discontinue et ouverte à une éventuelle rédemption. La partie consacrée à Benjamin traite des trois modèles de l'histoire dégagés par le philosophe : théologique, 
esthétique, historique, complémentaires et non antagonistes. Le premier, prédominant dans les * écrits de jeunesse *, oriente les deux essais, sur le langage et sur la traduction, que Mosès lit dans une continuité heuristique : là où la dégradation du langage symbolise la décadence de l'aventure humaine, la traduction indique la direction d'une restauration sous la forme d'une réalisation de virtualités présentes dès l'origine et soumises au devenir historique (1992, p. $100 \mathrm{sq}$.). Il s'agit cependant d'un examen ponctuel et succinct de la notion de traduction dans un schéma de pensée plus général alors qu'il est possible de voir dans la traduction un paradigme éclairant aussi bien la façon dont l'histoire s'offre à l'intellection que la propre méthode de Benjamin.

Françoise Proust interroge de même la philosophie de l'histoire de Benjamin comme axe central de sa pensée, de la littérature à la politique, et dans cette perspective consacre au langage un des six chapitres de son ouvrage l'Histoire à contretemps. Le temps historique chez Walter Benjamin. Cet examen est lié à la question de la vérité, de sa nature et de ses manifestations. La traduction joue ici un rôle exemplaire puisque l'essence de la vérité est de s'affirmer autoritairement, d'ellemême, en dehors de toute subjectivité et toute intentionalité. Or l'essai de Benjamin insiste sur l'autonomie du processus traductif en regard d'une quelconque soumission à un devoir de communication. Une telle décharge renforce ainsi une fonction cruciale de dévoilement de la vérité. * Au fur et à mesure que croît l'écart entre deux langues [...] croît l'écart entre la signification de l'œuvre conforme à l'intention de son auteur [...] et sa vérité [...]. Au fur et à mesure que décline et meurt la signification de l'œuvre, croît sa vérité, que seule révèle sa traduction. * (1994, p. 133) Ici encore, devant l'acuité et la qualité de la lecture (une des meilleures introductions à l'œuvre), on peut regretter que le concept benjaminien de traduction ne reçoive qu'une brève considération alors qu'il est reconnaissable, par exemple, dans ce que dit Françoise Proust de l'événement (ch. II), à la fois unique 
et reproduisible, ou des formes textuelles de la modernité (ch. VI), placées sous les signes de l'éclatement et de la survivance.

On regrettera encore la non-extension du concept dans l'ouvrage de Catherine Perret Walter Benjamin sans destin puisque, qu'elle traite de l'écriture, de la critique, de l'identité ou de l'histoire, elle insiste sur un permanent écart irrémédiable entre l'objet et la conscience dans l'approche benjaminienne. De surcroît, dans les quelques pages consacrées à la traduction, elle invite expressément le lecteur à ne pas se tromper sur le sujet de l'essai qu'elle compare à un « décor en trompe-l'œil ». Notant qu'il est " communément décrié ", sans doute parce qu'il s'en prend à l'immuabilité du sens et du langage, elle relève l'idée, révélée dans le langage, d'une " vie saisie par l'épreuve de la mort " (1992, p. 24) et définie par l'histoire. Le texte de Benjamin met surtout en lumière le travail traductif à l'intérieur des langues, entre les formes figées et leur capacité visionnaire initiale, qui marque leur liberté et leur développement historique. " En fait, et sous le couvert d'une théorie de la traduction, Benjamin dessine le projet d'une archéologie de la langue, remontant le procès historique des modes d'énonciation jusqu'à ce "parler magique" que les avancées successives de la rationalité auraient recouvert au profit de la dimension informative et instrumentale du langage. " (p. 26) S'il est vrai que l'essai déborde amplement la seule analyse du passage inter-linguistique, le concept même de traduction est loin d'être négligé dans l'ensemble de l'œuvre et peut se retrouver au contraire dans les autres interrogations de Benjamin, au service de ce projet archéologique si pertinemment dénommé.

Déception encore quand un lecteur aussi fin que Pierre Missac ne consacre qu'une phrase à \& la Tâche du traducteur " pour déplorer n'y point trouver a les principes ou les recettes d'une bonne traduction " (1987, p. 44), alors que le titre même du livre, Passage de Walter Benjamin, saisit une notion-clé de l'essai et que tout un chapitre traite de l'“ Homo scriptor " (pp. 49-92) sous les rubriques " Collecter, lire, écrire, jouer " (traduire ne s'imposait-il 
pas?).

Mention particulière, en revanche, doit être faite de l'article de Martine Broda dans le numéro de la revue Europe consacré à Walter Benjamin. Traductrice de Paul Celan, à l'cuvre réputée difficile, après avoir relevé chez les deux auteurs des affinités philosophiques en ce qui touche leur conception messianique, c'est-à-dire rédemptrice, de l'histoire, elle expose comment « la Tâche du traducteur " a pu l'aider dans son travail même, démentant le lieu commun à valeur de chef d'accusation voulant que la réflexion benjaminienne ne soit aucunement susceptible d'application pratique. Guidée par les principes d'" étrangéisation " et de littéralité, cette façon de traduire est, selon elle, " pour diverses raisons qui tiennent surtout à l'extrême précision de la poésie de Paul Celan et à son rapport très particulier, conflictuel, à la langue, la plus souhaitable ou même la seule possible " (1996, p. 182). La langue de Celan étant très spécifique, idiomatique, c'est une double étrangeté que sa version devait rendre : « celaniser le français encore plus que le germaniser " (ibid.), ce qu'elle a pu faire grâce au concept benjaminien de " ressemblance non sensible".

Tout autre est la direction empruntée par Rainer Rochlitz dans son étude de la philosophie de Benjamin puisque son ambition est de restituer une logique dans le foisonnement de ses écrits et d'y introduire une systématicité. Ce n'est pas le lieu de critiquer une approche qui pourrait sembler contraire à la méthodologie même de Benjamin et nous reconnaîtrons l'intérêt d'une section réservée à la théorie de la traduction dans la première des trois divisions de l'ouvrage (langage, art, histoire). L'exposé, d'une grande minutie, rapporte en les clarifiant les thèses benjaminiennes, notamment dans leur rapport aux théories du langage et de l'histoire en général. Sur deux points, cependant, la lecture de Rochlitz se fait critique. Le premier touche à la fonction de révélation de la vérité dévolue à la traduction. S'interrogeant sur la pertinence de ce rapprochement entre philosophie et traduction, et donc sur 
l'originalité même de la position de Benjamin qu'il vient si bien d'énoncer, Rochlitz se demande maintenant * si cette théorie rend compte de ce dont il s'agit dans la traduction qui a peut-être néanmoins à voir avec "Ies lecteurs qui ne comprennent pas l'original" et à qui il faut "communiquer" plus qu'un simple contenu discursif." (1992, p. 39) Dans un même mouvement régressif, il s'attaque ensuite à l'éclairage théologique que Benjamin donne à la manifestation esthétique, particulièrement quant à la nature de la littérarité des textes. " C'est parce qu'il rapproche verbe sacré et verbe poétique [...] que Benjamin peut parler emphatiquement du "contenu de vérité" des cuvres d'art [...]. Mais ce qui confère leur traductibilité aux grandes œuvres littéraires n'est pas leur vérité au sens strict, c'est leur qualité littéraire d'intensité au sens large et de cohérence signifiante [...]. . (p. 41) Mais ce que condamne, assez banalement, Rochlitz au nom d'une " raison profane " (p. 42), assurant à l'esthétique son domaine spécifique, définit précisément la tentative philosophique de Benjamin de sortir des impasses du rationalisme en brouillant les catégories épistémologiques. Interpréter Benjamin en termes kantiens ne tient-il pas alors d'une erreur de traduction?

On ne saurait reprocher à Jean-René Ladıniral de ne pas s'expliquer sur les raisons de sa sévérité critique et on souhaiterait parfois que les benjaminiens affichassent les mêmes ardeurs pour expliquer ou défendre l'essai. Sans compter les références éparses, deux articles (1981 et 1988) exposent avec précision sa lecture qu'il étaye d'arguments philosophiques aussi bien que nourris de sa pratique de traducteur de la philosophie allemande. Parmi divers reproches (écriture ésotérique, pensée hermétique, attachement à la forme et au littéralisme, défense de la valeur du mot) se dégage le principal grief édicté, l' $\propto$ anticommunicationalisme " articulé aux niveaux esthétique, traductologique et langagier. Or, en une double mise en perspective frisant la contradiction, une telle attitude est rapprochée à la fois de l'« antihumanisme et l'impersonnalisme de Heidegger * (1981, p. 72) et, avec insistance, de l'héritage * théologico-bibliste " hébraïque du judaïsme allemand. "Violence à la langue-cible " et " re-sacralisation de la littérature "(p. 74) en 
sont les conséquences négatives. Ainsi posée, la thèse de "l'inconscient théologique " (1988, p. 42) à l'œuvre sous ou derrière les théories de la traduction, que Ladmiral reprendra en divers endroits, débouche sur une distinction fortement polarisée entre l'irrationalisme d'un courant " sourcier " facilement reconnaissable comme rattaché à la tradition juive et la « rationalité positive » d'une " traductologie cibliste » qu'il défend, inspirée du catholicisme ( $p .43$ ). Outre le péché de réductionisme et l'idéologisation suspecte de l'analyse, c'est encore le souci historique de Benjamin qui est évacué.

Dans le camp adverse, si on se plaît à suivre la logique d'opposition prémentionnée, on trouvera celui qui fut l'antagoniste désigné, Henri Meschonnic dont la pensée traductologique se situe effectivement dans le sillage de celle de Benjamin, notamment par la place centrale accordée à l'historicité dans la pratique et la théorisation du traduire. On ne trouve cependant pas un traitement qui serait exclusivement et spécifiquement consacré au philosophe. Considérons Pour la poétique II qui contient de manière programmatique les thèses de Meschonnic; si Benjamin est cité ponctuellement (1986a, p. 300) dans la section finale, "Traduire ", de "Poétique du sacré dans la Bible n qui clôt la première partie consacrée à l'écriture, il ne l'est pas dans la seconde partie consacrée à la traduction. L'inspiration est cependant clairement benjaminienne comme en témoigne l'exergue d'une des sections (p. 327) ${ }^{3}$. Divers passages, néanmoins, attestent explicitement l'influence, traduite en termes appartenant immanquablement à Meschonnic : “ De même la tâche du traducteur est de produire un passage qui reste passage. Non, comme le veut l'idée courante, une arrivée, mais un rapport demeuré rapport. Il ne se termine pas en transport de langue. Il reste en chemin, pour faire sentir le

${ }^{3} \mathrm{C}$ 'est encore Benjamin qui offre la citation en exergue de la Rime et la vie (Verdier, 1989) : précisément, et significativement, la phrase de l'essai sur la traduction posant l'équation entre vie et histoire. 
chemin. " (1986b, p. 709) Ou encore : « La modernité dans les transformations en cours du traduire en est un exemple révélateur, dont le point de départ symbolique est "la Tâche du traducteur" de Walter Benjamin en 1923. Le rythme et l'oralité y jouent un rôle fondamental. Sans minimiser les résistances, la traduction fait apparaître que l'identité, au lieu de s'opposer à l'altérité, n'advient que par l'altérité. La relation d'opposition transformée en relation d'historicité, de métissage. "(1995, pp. 449-450). Le refus d'une logique dualiste du signe, le primat accordé au rythme et au discours trouvent naturellement leurs assises dans les choix épistémologiques dessinés par Benjamin, la fidélité fût-elle parfois critique, historicité oblige.

On a pu situer certains aspects de la réflexion d'Antoine Berman dans la même mouvance théorique. De fait, son dernier ouvrage, posthume, Pour une critique des traductions : John Donne, annonce un Traduire Benjamin à paraître, dont on ne peut que souhaiter la sortie effective. L'influence est reconnue à différents égards, débordant l'apport strictement traductologique pour appuyer le projet global de Berman : « Mais mon analyse des traductions, étant et se voulant une critique, se fonde également sur Walter Benjamin, car c'est chez lui qu'on trouve le concept le plus élevé et le plus radical de la critique "littéraire" - et de la critique tout court. Non seulement Benjamin est indépassable, mais il est encore en avant de nous. " (1995, p. 15) Il lui emprunte notamment les notions de " moment négatif " de la critique et de contribution à la " survie " de l'cuvre ainsi qu'un " principe de sobriété dans l'art " ( pp. 208 et 217). Par ailleurs, Benjamin est à plusieurs reprises cité comme jalon essentiel dans l'histoire et la constitution de la traductologie. Les références antérieures à Benjamin sont cependant plus ambigües. L'Épreave de l'étranger fonde la désormais familière " visée éthique * de la traduction (a ouvrir au niveau de l'écrit un certain rapport à l'Autre, féconder le Propre par la médiation de l'Étranger ", 1984, p. 16) contre - et non seulement par contraste - une " visée métaphysique de la traduction ", précisément associée à Benjamin et liée à une 
« pulsion du traduire * (p. 21), sans d'ailleurs que soit explicité le passage du philosophique au psychologique. « C'est la traduction contre Babel, contre le règne des différences, contre l'empiricité. * (p. 21) ${ }^{4}$ Ou encore : “ On pourrait dire que la visée métaphysique de la traduction est la mauvaise sublimation de la pulsion traduisante, alors que la visée éthique est son dépassement. " (p. 23) Outre l'étrangeté d'un lexique freudo-hégelien réénoncé en termes de morale, on peut se demander quels sont les passages de Benjamin méritant une telle interprétation. En revanche, dans un texte reprenant un séminaire de la même année, l'appréciation se module plus positivement : outre un rôle fondateur reconnu au philosophe dans le développement de la traductologie (1985, p. 39 $s q$ ), son essai est cité à plusieurs endroits (pp. 86-87 et 89-90) pour étayer deux fondements notionnels propres à dégager la dimension éthique de la traduction : le refus de réduire sa visée à la communication et la nécessité de considérer la "littéralité charnelle " du texte original dans l'exigence de fidélité.

" Traduire ", tel est le titre du texte consacré par Maurice Blanchot à l'essai et rien ne pouvait mieux signifier la justesse et l'élégance avec lesquelles le critique (au sens de Benjamin, c'està-dire dont la "tâche " doit conjuguer une double fécondité : commentaire et création, telle la traduction) a su rendre les thèses exprimées ${ }^{5}$. Ses lignes déclinent au plus près les deux aspects dont

\footnotetext{
${ }^{4}$ Voir, pour une vue exactement opposée, les citations de Blanchot infra.
}

${ }^{5}$ Un recueil antérieur contient un autre essai de Blanchot consacré à la traduction, \& Traduit de... ", qui, si Benjamin n'y est pas nommé, exprime certaines idées similaires : La traduction, si elle est bonne, apporte avec elle, sans recours à une incohérence factice, le sentiment d'un léger écart entre les mots et ce qu'ils visent, d'une possibilité pour eux, de glisser hors de cette forme qu'on leur a donnée pour retoumer à leur point de départ, qui est ici la langue originale mais qui symbolise aussi le fond originel sur lequel sont prélevés les mots à naître d'un langage qui se 
nous avons à plusieurs reprises souligné le manque dans les ouvrages précédemment traités : la révélation des différences dans le devenir historico-messianique des langues. « [Tout] traducteur vit de la différence des langues, toute traduction est fondée sur cette différence, tout en poursuivant, apparemment, le dessein pervers de la supprimer. [...] À la vérité, la traduction n'est nullement destinée à faire disparaître la différence dont elle est au contraire le jeu [...]. L'original n'est jamais immobile, et tout ce qu'il y a d'avenir dans une langue à un certain moment, tout ce qui en elle désigne ou appelle un état autre, parfois dangereusement autre, s'affirme dans la solennelle dérive des cuvres littéraires. La traduction est liée à ce devenir, elle le "traduit" et l'accomplit [...]. * (1985, pp. 70-71) Dans le jeu incessant entre identité et altérité où le traducteur gagne son titre de " maître secret de la différence des langues * (ibid.), Blanchot reconnaît ce que dans toute son œuvre il a situé au cœur de la littérature et qui se manifeste par le risque de la folie. Le recueil contenant l'essai de Blanchot sur l'essai de Benjamin s'intitule L'Amitié.

\section{Université de Montréal}

\section{RÉFÉRENCES}

BERMAN, Antoine (1984). L'Épreuve de l'étranger. Culture et traduction dans l'Allemagne romantique. Paris, Gallimard.

-(1985). « La traduction et la lettre ou L'auberge du lointain *. Les Tours de Babel. Mauvezin, Trans-Europ-Repress.

sépare à peine du vide. " ( La Part du feu, Paris, Gallimard, 1987; $1^{\circ}$ éd. : 1949, p. 184) 
-(1995). Pour une critique des traductions : John Donne. Paris, Gallimard.

BLANCHOT, Maurice (1985; $1^{\mathrm{c}}$ éd. : 1971). L'Amitié. Paris, Gallimard.

BRODA, Martine (1996). "Ce que Benjamin m'a apporté dans mon propre travail ", Europe, ${ }^{\circ}$ 804, pp. 180-184.

LAMY, Laurent (1993). " Du "traduire" comme extradition ", Discours social, vol. 5, n० 3-4, pp. 93-98.

-(1995). « Pas de deux : le mésocosme de la traduction comme matrice d'une sémantique frontalière ", Meta, vol. $40, \mathrm{n}^{\circ} 3$, pp. 461-477.

LADMIRAL, Jean-René (1981). " Entre les lignes, entre les langues ", Revue d'esthétique, nouvelle série, $\mathrm{n}^{\circ}$ 1, pp. 67-78.

-(1988). "Les enjeux métaphysiques de la traduction. À propos d'une critique de Walter Benjamin ", Le Cahier du collège international de philosophie, $\mathrm{n}^{\circ} 6$, pp. 39-44.

MAN, Paul de (1991). " Conclusions : "la Tâche du traducteur" de Walter Benjamin o (tr. par A. Nouss), TTR, vol. IV, $\mathrm{n}^{\circ}$ 2, pp. 21-52.

MESCHONNIC, Henri (1986a; $1^{\text {tre }}$ éd. : 1973). Pour la poétique II. Paris, Gallimard.

- (1986b). * L'Allégorie chez Walter Benjamin, une aventure juive ». Walter Benjamin et Paris. Paris, Cerf.

- (1995). Politique du rythme. Politique du sujet. Lagrasse, Verdier.

MISSAC, Pierre (1987). Passage de Walter Benjamin. Paris, Le Seuil. 
MOSÈS, Stéphane (1992). L'Ange de l'histoire. Rosenzweig, Benjamin, Scholem. Paris, Le Seuil.

NOUSS, Alexis (1993). " Ceci n'est pas une traduction ou Le péché de Babel ", Discours social, vol. 5, $\mathrm{n}^{\circ}$ 3-4, pp. 81-92.

-(1995). \& La traduction comme OVNI „, Meta, vol. 40, $\mathrm{n}^{\circ} 3$, pp. 335-342.

PERRET, Catherine (1992). Walter Benjamin sans destin. Paris, La Différence.

PROUST, Françoise (1994). L'Histoire à contretemps. Le temps historique chez Walter Benjamin. Paris, Cerf.

ROCHLITZ, Rainer (1992). Le Désenchantement de l'art. La philosophie de Walter Benjamin. Paris, Gallimard.

STEINER, George ([1975] 1978). Après Babel. Une poétique du dire et de la traduction (tr. Lucienne Lotringer). Paris, Albin Michel.

RÉSUMÉ : La réception de l'essai sur la traduction dans le domaine français - Cette recension critique rend compte d'une dizaine de titres ayant traité de l'essai sur la traduction de Walter Benjamin afin d'en présenter les grandes lignes de la réception dans le domaine français. L'auteur y montre comment, sauf exceptions, l'essai est souvent lu de manière partiale, restrictive ou coupé du reste de l'œuvre.

ABSTRACT : Reception of the Essay on Translation in French -Eleven studies in French of Walter Benjamin's essay on translation are examined here with a view to determining the way the essay has been received by French readers. The article demonstrates that all too often the essay has been read in a partial 
and biased manner, that its meaning has been restricted, and that the text has been divorced from Benjamin's other works.

Alexis Nouss : Département de linguistique et de traduction, Université de Montréal, C.P. 6128, Succursale Centre-Ville, Montréal (Québec) H3C 3J7. 